\title{
L'écotourisme terrestre en Polynésie française : Vers un renouveau touristique culturel ?
}

L'exemple du birdwatching

Jean-Marc Salducci

\section{OpenEdition}

\section{Journals}

Édition électronique

URL : http://journals.openedition.org/jso/184

DOI : $10.4000 /$ jso. 184

ISSN : $1760-7256$

Éditeur

Société des océanistes

Édition imprimée

Date de publication : 1 décembre 2004

Pagination : 185-187

ISSN : 0300-953x

Référence électronique

Jean-Marc Salducci, «L'écotourisme terrestre en Polynésie française : Vers un renouveau touristique culturel ? », Journal de la Société des Océanistes [En ligne], 119 | Année 2004-2, mis en ligne le 01 décembre 2007, consulté le 04 mai 2019. URL : http://journals.openedition.org/jso/184 ; DOI : $10.4000 /$ jso. 184 


\section{L'écotourisme terrestre en Polynésie française : Vers un renouveau touristique culturel ? L'exemple du birdwatching}

par

Jean-Marc SALDUCCI*

\section{RÉSUMÉ}

Le birdwatching au secours du tourisme local? De tels propos pourraient prêter à sourire, si la Polynésie française n'attirait pas déjà des birdwatchers du monde entier, alors qu'elle ne dispose encore d'aucune structure pour les accueillir. Portrait d'une activité potentielle, certes marginale, mais qui pourrait concourir au développement et à la diversification d'un écotourisme exagérément orienté vers le milieu maritime.

MoTS-CLÉS: écotourisme, birdwatching, richesse, endémisme, potentiel, sensibilisation, renouveau culturel, sauvegarde, patrimoine.

Aujourd'hui, la Polynésie française, dotée statutairement d'une autonomie de plus en plus étendue, se doit d'acquérir parallèlement les ressources économiques répondant à ces enjeux. Dans ce cadre, le tourisme apparaît plus que jamais comme le fer de lance de son économie. Il est vrai que le mythe du paradis des mers du Sud contribue largement au succès touristique local. Cependant, le marché mondial sur ce secteur apparaît de plus en plus concurrentiel et la Polynésie française, pour assurer sa place dans cette dynamique et parce qu'elle souhaite cibler une

\section{ABSTRACT}

Can birdwatching help local tourism? Such remarks could rise a smile if French Polynesia didn't already attract birdwatchers from the whole world even if it doesn't have any reception facilities yet. This article presents a picture of a potential but certainly marginal activity, which could work towards the expansion and diversification of an ecotourism excessively turned towards the maritime environment.

KEYwORDs: ecotourism, birdwatching, wealth, native species, spring culture, preservation of heritage.

frange touristique à hauts revenus, se doit d'afficher plus que jamais une qualité irréprochable pour son environnement, tout en tenant compte de l'évolution des aspirations et de la demande. Ainsi a-t-elle intégré tout naturellement le phénomène écotouristique, au regard des potentialités réelles qu'elle détient dans ce domaine: plongée sous-marine (archipels de la Société, des Tuamotu, des Marquises) et observation des baleines à bosse (archipel des Australes) ne représentent que quelques exemples parmi bien d'autres. S'il est indéniable que l'écotourisme

\footnotetext{
* Professeur certifié au lycée d'Uturoa, DEA Espaces et sociétés du monde océanien sur la relation entre les hommes et leurs
} oiseaux en Polynésie française, imsalducci@yahoo.fr 
local attire un nombre de touristes important, force est de constater qu'il est essentiellement orienté vers le milieu marin. Cela reviendrait-il à dire que le milieu terrestre ne peut offrir les mêmes potentialités? Au contraire!

Tout comme le milieu marin, le milieu terrestre présente une richesse tout à fait remarquable : les paysages y sont magnifiques et la biodiversité d'une grande qualité, tant au niveau faunistique que floristique. Pourtant, mis à part quelques safaris $4 \times 4$, on ne peut que déplorer la quasi-absence des activités proposées aux touristes dans ce domaine. La randonnée pédestre, un argument touristique "phare» de l'île de $\mathrm{La}$ Réunion, n'est ici qu'embryonnaire et généralement pratiquée par des résidents, faute d'informations diffusées dans les hôtels ou les pensions, malgré un potentiel remarquable : les amoureux de la nature doivent donc se débrouiller par euxmêmes pour découvrir le patrimoine naturel local. Cela est d'autant plus regrettable que, sur certains points, la Polynésie française dispose de ressources tout à fait exceptionnelles. À titre d'exemple, elle constitue un arrêt quasi-obligé dans la région Pacifique pour les birdwatchers (Mапи 19, 1997 : 3) : il s'agit de touristes généralement anglophones dont l'activité (pratiquée couramment aux États-Unis et en NouvelleZélande) consiste à observer un maximum d'espèces d'oiseaux.

Cette activité s'étend aujourd'hui à l'ensemble de la planète. Or, il s'agit d'une frange touristique intéressante pour deux raisons principales. Tout d'abord, elle dispose généralement de moyens élevés. Ainsi, des croisières de birdwatchers sont organisées plus ou moins régulièrement au départ de Papeete et un groupe de ces touristes a déjà, moyennant finances, dérouté le navire Aranui (qui ravitaille habituellement les îles Marquises) vers un atoll isolé des Tuamotu dans le seul but d'y apercevoir le tìtī (ou bécasseau polynésien), un limicole endémique qui ne survit plus que sur quelques atolls (Fontaine et al., 1999 : 40). De plus, le birdwatcher, parce qu'il recherche des espèces endémiques et rares, n'hésite pas à séjourner dans des îles isolées, concourant ainsi à la mise en valeur de leur potentialité touristique. Si les birdwatchers sont encore relativement peu nombreux à venir chaque année, il est indéniable que la Polynésie française représente une attractivité certaine : si la majorité d'entre eux provient des États-Unis et de Nouvelle-Zélande, il en arrive aussi d'Europe, voire même de Singapour! Pourquoi un tel attrait ? Parce que la Polynésie française n'abrite pas moins de quinze espèces et quarante sousespèces d'oiseaux endémiques (Thibault et
Guyot, 1988: 87), dont certaines figurent parmi les plus rares au monde! Elle dispose donc d'un véritable trésor ornithologique à promouvoir.

Il se pourrait pourtant que cette activité touristique ne puisse jamais être développée, en dépit de premiers résultats encourageants. D'une part, parce que de nombreuses espèces d'oiseaux sont aujourd'hui menacées de disparition à court ou moyen terme (Thibault et Guyot, 1988 : 106) : à titre d'exemple, le 'ômāma'o ou monarque de Tahiti ne compte plus qu'une quarantaine d'individus (Blanvillain et al., 2002: 197), tout comme le kote'ute'u ou martin-chasseur des Tuamotu, dont les effectifs ont chuté pour ne représenter aujourd'hui qu'une cinquantaine de spécimens (Gouni et al., 2004 : 30). D'autre part, parce que, faute de la connaître, la population locale n'est pas (ou peu) sensibilisée à la préservation de son avifaune. Pour preuve, les noms vernaculaires des animaux marins sont encore couramment employés, alors que ceux des oiseaux sont souvent oubliés : qui connaît le nom tahitien du monarque? Cette situation résulte d'une pensée liée à la notion d'intérêt pratique. Les poissons, par exemple, constituent une source d'approvisionnement en nourriture : ils sont en cela « utiles » et, de ce fait, généralement bien connus de la population. Les oiseaux, en revanche, sont négligés parce qu'ils ne jouent plus aucun rôle dans la société actuelle. Pourtant, à l'époque pré-européenne, ils étaient hautement considérés, notamment parce qu'ils servaient d'intermédiaires entre le monde des hommes et celui des dieux (Salducci, 2002 : 57). On cherchait alors à les attirer pour communiquer avec les divinités et on prêtait la plus grande attention à l'interprétation de leurs chants, annonciateurs de bons ou mauvais augures : leur importance dans la vie sociale leur permettait donc d'être reconnus. Aujourd'hui, seuls certains oiseaux de mer sont encore dénommés selon leur appellation vernaculaire, car ils restent utiles dans le domaine de la pêche (Salducci, 2002: 89). Les espèces terrestres, quant à elles, ont en quelque sorte déjà disparu car, faute de rôle, elles ont été progressivement oubliées. De plus, cette évolution des mentalités, accentuée par l'urbanisation du style de vie, a provoqué également un appauvrissement culturel, (Salducci, $2002: 100$ ) car cet oubli des oiseaux s'est accompagné d'un oubli des légendes et croyances afférentes au monde aviaire polynésien. De ce fait, comment peut-on envisager une protection efficace d'espèces locales si elles se sont déjà éteintes dans la conscience collective? Il faut donc préalablement redonner au patrimoine aviaire une place 
dans la société humaine si l'on veut espérer un soutien de la population à son égard.

Ainsi, réhabiliter les oiseaux polynésiens reviendrait à faire d'une pierre «trois» coups : en leur attribuant un rôle (touristique) et donc un intérêt, les oiseaux pourraient permettre l'attraction des birdwatchers, une redécouverte d'ordre culturel, mais surtout la protection de l'avifaune locale. Des opérations de sauvegarde sont aujourd'hui menées par la Société d'ornithologie de Polynésie Manu, avec l'appui du gouvernement local, mais ces opérations ne pourront être couronnées de succès qu'avec le concours des populations.

C'est pourquoi l'essor du birdwatching est intéressant à plus d'un titre. Il permet tout d'abord de développer un secteur touristique et de participer ainsi à l'essor d'îles isolées par la création d'emplois directs et indirects, sans pour autant nécessiter des investissements importants (formation de guides dans les îles potentiellement intéressantes). Il sensibilise la population envers la préservation de son patrimoine naturel terrestre et concourt ainsi à la pérennité de sa richesse. Il représente enfin un atout culturel en favorisant la redécouverte et la sauvegarde des récits et légendes liés à ce patrimoine. Alors, le birdwatching, un concept touristique prometteur ? Certaines îles des Marquises l'ont d'ores et déjà pris en compte et cherchent à le promouvoir : à Nuku-Hiva, on emmène parfois les visiteurs à la rencontre du upe (carpophage des Marquises), l'un des plus gros pigeons du monde, tandis que l'île de Ua-Huka valorise son image d'éden pour les oiseaux endémiques marquisiens...
Certes, une telle activité ne représente sans doute pas un intérêt globalement majeur pour l'avenir du marché touristique local, mais à l'heure où la nécessité de se démarquer dans ce secteur est plus que jamais à l'ordre du jour, il serait dommage de ne pas encourager un attrait qui ne demande qu'à être connu...

\section{BIBLIOGRAPHIE}

Blanvillain Caroline, Jean-Marc Salducci, Gaston TUtURURA et HAND, 2002. Impact of introduced birds of the recovery of the Tahiti flycatcher Pomarea nigra, critically endangered forest bird of Tahiti, Biological conservation 109, pp. 197-205.

$M_{A N U}$ 1990-2003, Bulletin de la Société d'ornithologie de Polynésie, Papeete.

Fontaine Pierre, Odile et Jacques Fossati, Véronique Mu-Liepman, Philippe Raust et Yolande VernauDON, 2000. Manu, Les Oiseaux de Polynésie, Papeete, Au-vent-des-îles, collection Survol, 112 p.

Gouni Anne, Christophe NoIret, Tetai TeHEI et JeanBaptiste TAHUA, 2004. Étude du Martin-chasseur de Niau, Todiramphus gambieri niauensis. Mise en place d'un programme de conservation, Papeete, Société d'Ornithologie de Polynésie Manu, $44 \mathrm{p}$.

SALDUCCI Jean-Marc, 2002. L'oiseau en Polynésie française : héritages du passé et nécessités contemporaines de préservation, DEA Espaces et sociétés du monde océanien: dynamiques contemporaines, Papeete, université de Polynésie française, 165 p.

Thibault Jean-Claude et Isabelle GuYot, 1988. Livre rouge des oiseaux menacés des régions françaises d'outre-mer, Conseil international pour la protection des oiseaux, monographie 5, $258 \mathrm{p}$. 